\title{
Should states adopt newborn screening for early infantile Krabbe disease?
}

\author{
David P. Dimmock ${ }^{1-4}$
}

Infantile-onset Krabbe disease (EIKD) is a progressive neurological disorder. By the time children are symptomatic, there is no effective therapy. For those of us who have watched the decline of children afflicted by this disorder, it is heart-wrenching. For parents, the feeling of powerlessness and the suggestion that an earlier diagnosis may have improved the outcome lead to the desire to prevent other parents from suffering in this way. Consequently, it is logical to wish to identify this disorder with newborn screening (NBS) and offer the hope of potential therapy. However, to add another condition to a state or federal NBS panel requires us to consider the potential harms of NBS as well as the benefits-not only to those affected with a condition but also to other families who may receive false-positive test results or the knowledge of a later-onset disease. In addition, a program must take into account the opportunity cost of spending resources on screening one condition versus another or of spending time and money on other public health initiatives.

Over the past decade, several states have added EIKD screening to their NBS by legislative mandate. ${ }^{1}$ The article in this issue of Genetics in Medicine provides an important update on the technical success of the largest cohort of individuals undergoing such mandated screening. ${ }^{2}$ As such, it is a valuable source of information on the real-world effects of such deployment and contributes significant new data compared with that reviewed just over 6 years ago by what is now known as the US Department of Health and Human Services' Advisory Committee on Heritable Disorders in Newborns and Children. ${ }^{3}$

In Wisconsin, a framework for evaluating the addition or deletion of testing was proposed to the Wisconsin Secretary of Health in June 2013 (Report to the Secretary of the Wisconsin Department of Health Services: Newborn Screening Task Force, June 2013). Wisconsin is currently considering the addition of EIKD screening to its NBS panel. As part of this consideration, I reviewed the previous work of the federal advisory committee and more than 1,000 new abstracts. In preparing this Commentary, I also reviewed the article by Orsini et al. ${ }^{2}$ in this issue. A summary of these findings is compared with the "Wisconsin addition criteria" to evaluate the implications these new data have for the decision as to whether to adopt or remove NBS for EIKD.

\section{CRITERIA FOR IMPLEMENTATION OF NBS}

Criterion 1: the disorder causes serious health risks in childhood that are unlikely to be detected or prevented in the absence of NBS

Before the introduction of NBS in New York, childhood-onset Krabbe disease was estimated to account for $90 \%$ of symptomatically identified individuals with Krabbe disease. Most present with progressive neurological symptoms before the age of 6 months. Symptomatic presentation prior to NBS was estimated to occur in 1/100,000 infants, but data from New York and Missouri have suggested that the real incidence is closer to 1:400,000 (ref. 1). Without intervention, individuals who present clinically with infantile Krabbe disease will die before the age of 6 years. There are clear data indicating that at the time that children are significantly symptomatic therapeutic intervention (hematopoietic stem cell transplantation, HSCT)) is not effective. There is no evidence to date that NBS increases the rate of detection of EIKD. However, the article by Orsini et al. $^{2}$ demonstrates that NBS will identify these children in the presymptomatic phase, potentially enabling therapy prior to symptom onset.

\section{Criterion 2: the incidence, morbidity and mortality, and natural history of the disorder are known}

The natural history is well described for clinically identified individuals with Krabbe disease (Hunters Hope registry data).,

The original evidence review in 2009 concluded that there is poor genotype-phenotype correlation ${ }^{5}$ other than homozygosity of the $30-\mathrm{kb}$ deletion, which is strongly predictive of EIKD. Low levels of galactocerebrosidase activity ${ }^{4,6}$ do not entirely predict the age of symptom onset or severity of white matter changes. Subsequent conclusions from the registry suggest "The later onset Krabbe phenotypes differ from those with early infantile disease, but no specific predictor of phenotype was identified." In addition, the authors of the New York State program concluded that higher galactocerebrosidase activity was predictive of later symptom-onset times $(P=0.0011)$ but did not predict survival after symptom onset $(P=0.9064)$ when controlling for the logarithm of age at onset. ${ }^{8}$ This has been confirmed by further studies, ${ }^{8}$ with the authors concluding

${ }^{1}$ Human Molecular Genetics Center, Medical College of Wisconsin, Milwaukee, Wisconsin, USA; ${ }^{2}$ Division of Genetics, Department of Pediatrics, Medical College of Wisconsin, Milwaukee, Wisconsin, USA; ${ }^{3}$ Metabolic Subcommittee, State of Wisconsin Newborn Screening Program, Madison, Wisconsin, USA; ${ }^{4}$ Genetics Center, Children's Hospital of Wisconsin, Milwaukee, Wisconsin, USA. Correspondence: David P. Dimmock (ddimmock@mcw.edu)

Submitted 5 January 2016; accepted 8 January 2016; advance online publication 4 February 2016. doi:10.1038/gim.2016.6 
that "No correlation was found between enzymatic activity, onset age and disease progression" and "current knowledge about onset age, residual enzyme activity and molecular analysis still fail to allow the identification of patient candidates for treatment."

The new data ${ }^{2}$ continue to show that many children who fail the enzymatic test, whether false positives or EIKD cases, have previously undescribed variants of unclear clinical significance. This important article provides very useful information on genotypes associated with "pseudodeficiency" or late-onset disease, reducing by almost half the potential number of children who need to be followed if identified as a result of abnormal enzymatic screening. Nonetheless, even with this wealth of information, in the New York State experience another nine cases were left enzymatically defined as "high risk" without a clear diagnosis, ${ }^{2}$ potentially exposing these families to significant ongoing anxiety and uncertainty and the children to invasive testing.

\section{Criterion 3: conditions identified by NBS should be linked to interventions that have been shown in well-designed studies to be safe and effective in preventing serious health consequences}

As is typical for orphan diseases, there are no randomized controlled trials of therapeutic intervention. Instead, studies aiming for US Food and Drug Administration approval have typically been based on a comparison of historical or registry controls versus an intervention group. Escolar et al. reported that early HSCT offers advantages. The authors compared the outcomes of 11 asymptomatic newborns and 14 symptomatic infants with Krabbe disease who received HSCT. The survival rate was 100\% among the asymptomatic newborns and $43 \%$ among the symptomatic infants. Although cognitive function and language skills continued to develop appropriately in patients who underwent HSCT before symptom onset, gross motor impairment was noted even in some of these patients. ${ }^{9}$ Subsequently, a single case report from Japan has demonstrated a similar good outcome. ${ }^{10}$ The long-term data from the New York program presented by Orsini et al..$^{10}$ have not demonstrated that early detection through NBS and early transplantation prevent neurological problems. Although the two surviving children who underwent transplantation are thought to be better neurologically than they would have been had they followed the typical fulminant course of EIKD, they have shown neurological progression. An earlier workshop reported that all infants with presymptomatic Krabbe disease transplanted at Duke University to date had developed neurological and other deficits, many of them severe. ${ }^{11}$

Concerning the peripheral neuropathy, Orchard and Tolar ${ }^{12}$ noted in 2010, "Their significant motor limitations are likely at least in part to be due to peripheral nerve demyelination, as is observed in the twitcher mice, a model for globoid cell leukodystrophy."

The new data from New York demonstrate dismal outcomes, with two of five patients dying as a result of complications from therapy prompted as a result of the abnormal newborn screen, one of five cases following the natural history of the disorder (the parents declined HSCT), and two of five children showing ongoing neurological decline despite receiving a transplant. ${ }^{2}$ It is difficult to compare these outcomes with that expected without transplant because there are no natural-history control data following a positive newborn screen. However, given the high early mortality, it seems likely that early detection by NBS has reduced the aggregate survival time of EIKD. The authors provide no quality of life data, but initiation of the conditioning required for stem cell transplantation is likely to have worsened the premortem quality of life in the fatal cases, suggesting that, in addition to reducing survival time, NBS for EIKD may worsen the quality of life for both the child and the family.

\section{Criterion 4: the interventions should be reasonably available to affected newborns}

As Steiner ${ }^{13}$ noted, "At best, treatment is effective in preventing severe cognitive deterioration but progression of some aspects of the disease continues nonetheless in some patients; at worst, transplant is unproven and experimental."

Most insurance excludes experimental or unproven treatment. Stem cell transplantation may not be covered by insurance unless a state mandates coverage at the same time as introducing NBS.

\section{Criterion 5: appropriate follow-up should be available for newborns who have a false-positive newborn screen}

The estimates for New York State presented by Orsini et al. ${ }^{2}$ suggest that the chances of having a "high-risk" white blood cell assay with an abnormal screen was $4 \%$, but only $1.4 \%$ were confirmed to have EIKD. This $2.6 \%$ discrepancy provides significant challenges for the NBS follow-up program. Specifically, there is an ongoing challenge establishing when a case has a false-positive result by any further functional means. Although most symptomatic patients with the early infantile phenotype manifested abnormal cerebrospinal fluid protein, magnetic resonance imaging, brainstem-evoked responses, and nerve conduction velocity studies, studies in affected children may have normal results. ${ }^{7}$ It is therefore challenging to exclude a diagnosis with an abnormal screen. Indeed, two children with high-risk enzyme levels were reported to be normal at 8 and 16 months of age. ${ }^{11}$ It is possible that psychosine levels may allow for further stratification in the future, ${ }^{1}$ but Orsini et al. ${ }^{2}$ report that nine children are being followed with "high-risk" enzymatic testing without a clear diagnosis.

All states currently screening or piloting screening use an enzymatic method as the initial step, followed by molecular methods (DNA testing). As noted above, enzymatic testing has a very poor positive predictive value, with 20 infants identified as positive for every confirmed case of Krabbe disease. Turgeon et al estimate that $5-15 \%$ of patients with abnormal screens will undergo extensive studies, including brain magnetic resonance imaging scans, lumbar puncture to obtain cerebrospinal fluid, and painful nerve sequencing conduction velocity studies, as well as brain stem auditory-evoked potentials and visualevoked potentials. ${ }^{1}$ 
Criterion 6: the characteristics of tests mandated for the newborn population should be known, including specificity, sensitivity, and predictive value

In 2006, New York State began screening all newborns for Krabbe disease. In this update on their experience, Orsini et al. ${ }^{2}$ provide a significant amount of new information on the characteristics of enzymatic testing. They demonstrate that the positive predictive value of an abnormal enzymatic test on dried blood spot screening is $1.4 \%$. Over the past 7 years, the New York State program has made significant advances in the ability to reduce the falsepositive rate. This article demonstrates that molecular testing can reduce the rate of false-positive results by almost half. However, the authors identified almost twice as many patients (nine versus five) who had abnormal white blood cell enzymatic testing results but the final diagnosis is not yet resolved as compared with confirmed positive cases. Only subsequent longer-term follow-up will determine whether the at-risk children described by Orsini et al. ${ }^{1}$ will develop symptoms at a later date, whether they are carriers of the disease, or whether they simply harbor nonpathogenic galactocerebrosidase-lowering polymorphisms.

Over the past decade, several important papers have shown that there is no correlation between enzymatic activity and age of onset of disease and that, aside from the common deletion, there is no concrete correlation between genotype and phenotype. ${ }^{13-15}$ Orsini et al. ${ }^{2}$ have now closed this gap by providing more concrete answers for some of the more commonly encountered pseudo-deficiency alleles.

The author is unaware of any programs planning to implement screening for the common deletion as a primary screen. This is probably because the false negative rate would be unacceptably high. The sensitivity and specificity of DNA sequencing in the context of NBS for Krabbe disease have not been published. However, the molecular data provided by Orsini et al. ${ }^{2}$ will improve the precision of DNA based diagnosis.

The evaluation of psychosine levels on dried blood spots is currently being considered. However, it is not feasible as a primary screen because it has a 17 -minute run time. ${ }^{1}$ The sensitivity and specificity of psychosine in the context of NBS have not been determined, and the article by Orsini et al. ${ }^{2}$ does not add to the information about this potential screen.

\section{Criterion 7: if a new sample-collection system is needed} to add a disorder to an NBS panel, then reliability and timeliness of sample collection must be demonstrated Orsini et al. ${ }^{2}$ show that a relatively robust signal, albeit with reduced specificity compared with a white blood cell assay, is possible from a normally collected neonatal dried blood spot.

\section{Criterion 8: before a disorder is added to the panel, the} details of reporting, follow-up, and management must be completely delineated, including development of standard instructions, identification of consultants, and identification of appropriate referral centers throughout the state/region Brochures for parents have been developed by New York, Illinois, and Missouri that could be modified by a state if a decision is made to proceed. Children will need to be referred to a center equipped with appropriate neurological services that can perform nerve conduction velocity studies. ${ }^{1}$ These centers will also need to be able to provide pretest counseling and preauthorization for molecular testing if not provided as part of the program, as well as to provide or refer for HCST.

\section{Criterion 9: recommendations and decisions should consider the costs of the screening test, confirmatory testing, accompanying treatment, and counseling, and the consequences of false positives; the mechanism of funding those costs should be identified; and expertise in economic factors should be available to those responsible for recommendations and decisions}

Screening test. The addition of EIKD screening would require the addition of extra equipment and personnel to a screening program. The exact costs will depend on whether the state adopted the tandem mass spectrometry method used in Illinois and New York or a microfluidic solution. New York has published costs of approximately $\$ 4$ per screening card. ${ }^{3}$

Follow-up of positive screens. Approximately $10 \%$ of patients with positive screens will undergo serial nerve conduction studies, magnetic resonance imaging scans, lumbar punctures, and neurological examinations. This is likely to be covered by insurance. ${ }^{1,6}$

Treatment. The costs of stem cell transplantation and immunosuppression are significant. These may be covered by the patient's insurance or by research protocols.

\section{SUMMARY}

The published experience to date regarding implementing NBS for EIKD has been dramatically enhanced by the data presented in this issue by Orsini et al. ${ }^{2}$ Although much has been written about the potential harms to individuals with an abnormal NBS for EIKD who do not have EIKD, several commentators have also asked whether screening provides a benefit when it leads to early identification of the disorder.$^{16}$ Orsini and colleagues' data suggest that the state-mandated, multimillion-dollar NBS program for EIKD in New York has failed to provide significant benefit to children with EIKD. Indeed, in addition to the potential harm to families receiving false-positive test results, NBS for EIKD appears to have resulted in a reduction in survival in individuals who have the disease. The data from the New York program suggest that NBS for EIKD should be abandoned, pending the development of improved screening or therapies shown to confer both survival and quality-of-life benefits over supportive care. The results of this experience suggest that research efforts should be focused on improving presymptomatic treatment outcomes in children identified by NBS prior to the redeployment of mandatory presymptomatic screening.

\section{ACKNOWLEDGMENTS}

D.P.D. wrote this Commentary in his personal capacity. The views expressed are his own and do not necessarily represent the views 
of the Medical College of Wisconsin, Children's Hospital of Wisconsin, or the State of Wisconsin Newborn Screening Program.

\section{DISCLOSURE}

The author declares no conflict of interest.

\section{REFERENCES}

1. Turgeon CT, Orsini JJ, Sanders KA, et al. Measurement of psychosine in dried blood spots-a possible improvement to newborn screening programs for Krabbe disease. J Inherit Metab Dis 2015;38:923-929.

2. Orsini JJ, et al. Newborn screening for Krabbe disease in New York State: the first eight years' experience. Genet Med 2016;18:240-249.

3. Kemper AR, Knapp AA, Green NS, Comeau AM, Metterville DR, Perrin JM. Weighing the evidence for newborn screening for early-infantile Krabbe disease. Genet Med 2010;12:539-543.

4. Duffner PK, Jalal K, Carter RL. The Hunter's Hope Krabbe family database. Pediatr Neurol 2009;40:13-18.

5. Tatsumi N, Inui K, Sakai N, et al. Molecular defects in Krabbe disease. Hum Mol Genet 1995;4:1865-1868.

6. Duffner PK, Caggana M, Orsini JJ, et al. Newborn screening for Krabbe disease: the New York State model. Pediatr Neurol 2009;40:245-252; discussion 253-255.

7. Duffner PK, Barczykowski A, Kay DM, et al. Later onset phenotypes of Krabbe disease: results of the world-wide registry. Pediatr Neurol 2012;46:298-306.
8. Jalal K, Carter R, Yan L, Barczykowski A, Duffner PK. Does galactocerebrosidase activity predict Krabbe phenotype? Pediatr Neurol 2012;47:324-329.

9. Escolar ML, Poe MD, Provenzale JM, et al. Transplantation of umbilical-cord blood in babies with infantile Krabbe's disease. N Engl J Med 2005;352: 2069-2081.

10. Yagasaki $H$, Kato $M$, Ishige $M$, Shichino $H$, Chin M, Mugishima H. Successful cord blood transplantation in a 42-day-old boy with infantile Krabbe disease. Int J Hemato/ 2011;93:566-568.

11. Duffner PK, Caviness VS Jr, Erbe RW, et al. The long-term outcomes of presymptomatic infants transplanted for Krabbe disease: report of the workshop held on July 11 and 12, 2008, Holiday Valley, New York. Genet Med 2009;11:450-454.

12. Orchard PJ, Tolar J. Transplant outcomes in leukodystrophies. Semin Hematol 2010;47:70-78.

13. Steiner RD. Commentary on: "Newborn screening for Krabbe Disease: the New York state model" and "the long-term outcomes of presymptomatic infants transplanted for Krabbe disease. A report of the workshop held on July 11 and 12, 2008, Holiday Valley, New York." Genet Med 2009;11:411-413.

14. Wenger DA, Luzi P, Rafi MA. Krabbe disease: are certain mutations diseasecausing only when specific polymorphisms are present or when inherited in trans with specific second mutations? Mol Genet Metab 2014;111: 307-308.

15. Wenger DA. Krabbe disease. In: Pagon RA, Adam MP, Ardinger HH, et al. (eds). GeneReviews ${ }^{\circledR}$. University of Washington: Seattle, WA, 1993.

16. Lantos JD. Dangerous and expensive screening and treatment for rare childhood diseases: the case of Krabbe disease. Dev Disabil Res Rev 2011;17:15-18. 